\title{
Compaction of Drosophila histoblasts in a crowded epidermis is driven by buckling of their apical junctions.
}

${ }_{4}$ Annafrancesca Rigato ${ }^{1,2^{*}}$, Huicheng Meng ${ }^{1}$, Faris Abouakil ${ }^{1}$, and Loïc LeGoff ${ }^{1 *}$

${ }_{5}^{1}$ Aix Marseille Univ, CNRS, Centrale Marseille, Institut Fresnel, Turing Center for Living Systems, Marseille, France

${ }^{2}$ Aix Marseille Université, CNRS, IBDM, UMR 7288, Marseille, France

*correspondance: rigato@fresnel.fr, loic.le-goff@univ-amu.fr

\& ABSTRACT

In many proliferating epithelia, cells present a polygonal shape that results from tensile forces of the cytoskeletal cortex and from the packing geometry set by the cell cycle ${ }^{1,2}$. In the larval Drosophila epidermis, two cell populations, histoblasts and larval epithelial cells, compete for space as they grow on a limited body surface. They do so in the absence of cell divisions. Here we show that histoblasts, which are initially polygonal, undergo a dramatic morphological transition in the course of larval development. Histoblasts change from a tensed network configuration, with straight cell outlines at the level of adherens junctions, to a highly folded morphology. The apical surface of histoblasts shrinks while their growing adherens junctions fold.

9 Volume increase of growing histoblasts is accommodated basally, compensating for the shrinking apical area. The folded geometry of apical junctions is reminiscent of elastic buckling. In accordance, we show that folding of junctions results from an imbalance between the growth of the junctions and the increasing crowding of the epidermis. The process also correlates with a change in the junctional acto-myosin cortex and possibly mechanical properties.

We propose a model in which crowding of the epidermis imposes a compressive load on the growing junctions which induces their buckling. Buckling effectively compacts histoblasts at their apical plane and may serve to avoid physical harm to these adult epidermis precursors during larval life. Our work also indicates that in growing non-dividing cells, compressive forces, instead of tension, may drive cell morphology.

\section{Introduction}

Morphogenesis proceeds through the mechanical interaction of cells in order to shape tissues. Our understanding of the cellular and subcellular processes that generate physical forces instructive for development has considerably improved in recent years ${ }^{3-6}$. At the single cell scale, the role of the cytoskeleton and of adhesion complexes in setting morphological changes has been well established ${ }^{4,7,8}$. As adhesion links cells to their environment, be it other cells or the extracellular matrix, the shape of a cell is determined both by its internally generated active forces and by environmental constraints ${ }^{9}$. Growth can act as a potent environmental constraint to shape cells and tissues. Spatial variations in the orientation or the rate of growth, sometimes called incompatible growth ${ }^{10,11}$ lead to mechanical pre-stress impinging upon cells. For example, if one or a patch of cells grows more than its surroundings, the overgrowing patch will be compressed and the surroundings will be stretched ${ }^{6}$.

Because of their biological relevance and ease of imaging, epithelial tissues have been particularly well characterized in terms of mechanics. The combination of modeling, analysis of cell shape and mechanical perturbations has led to the following understanding of epithelial mechanics: I) the tissue is in a tensed state; II) cell growth is usually balanced over the cell cycle (cells double in volume from beginning to end of the cell cycle); III) stress can be released through topological transitions such as cell neighbor exchange and oriented cell divisions ${ }^{12}$. With these elements, models were developed for epithelial tissues, able to capture many features of morphogenesis 2,13 . Epithelial monolayers are then described as tensed networks, formed by polygonal-shaped cells with straight borders ${ }^{14,15}$. Nevertheless, tensed epithelia that respect the characteristics above are only a small portion of the complex scenario of morphogenesis. Most of the time, a homogeneous population is considered, constituted of a single cell type.

Other than tensile forces, compressive forces are also important shape generators. For example, an elastic body under compressive forces can go through buckling instability ${ }^{16}$, a process at play in gut vilification ${ }^{17}$ or in the formation of brain cortical folds by differential growth of apposed cortical cell layers ${ }^{18}$. While these examples are taken from phenomena at large scale, in this work we find evidences that similar effects exist at the level of individual cells.

The epidermis of the Drosophila larva consists of two cell populations: the larval epithelial cells (LECs), which are 
large polyploid cells and the histoblasts, which are the precursors of the adult epidermis. These two cell populations form a continuous cell monolayer ${ }^{19}$. Histoblasts are clustered in nests of a fixed cell number ( 5 to 17 cells per nest) surrounded by LECs. Growth of both histoblasts and LECs happens without cell division over a large time span of larval development, from 4 hours after hatching until the pupal stage ${ }^{19,20}$. Histoblasts do not exchange neighbors during this period. The growth rates of histoblasts and LECs are different, with LECs increasing in volume about 150-fold during larval life, and histoblasts 60 -fold ${ }^{19}$. The larval body stops growing around 90 hours after egg laying (h AEL) ${ }^{19,21}$ while the epidermal cells continue to grow. Thus, this binary cellular system, where two cell populations grow and compete for space in the absence of stress-releasing topological transitions is likely to present a mechanical regime yet unexplored by other epithelial model systems.

Here, we investigate the shape of developing histoblasts. We developed a protocol for time-lapse imaging of individual cells throughout larval stages. We observed that histoblasts go through a considerable morphological transition between $90 \mathrm{~h}$ and $110 \mathrm{~h}$ AEL, changing from a tensed network configuration with straight cell outlines to a highly folded morphology of cell shapes, suggestive of compressive forces. We show that the formation of folded junctions is linked to the competition for space of LECs and histoblasts in a non-autonomous fashion, and is helped by a change in mechanical properties of the junctions. We propose a model according to which crowding of the epidermis imposes a compressive load on the growing junctions which induces their buckling.

\section{Results}

\section{Histoblasts undergo a dramatic morphological change during the L3 stage}

To investigate the growth and morphology of histoblasts during the last larval phase, we optimized a protocol for live imaging of the larval epidermis (see methods and Fig. S1). Briefly, larvae were anesthetized with Desflurane to prevent muscle contractions and oriented to image the histoblasts. After imaging, larvae were put back in soft medium, necessary for normal growth, at $25^{\circ} \mathrm{C}$. Recovery from anesthesia takes few minutes, and larvae can then develop normally. By repeating such procedure every few hours, we could image the same cells over several hours, without affecting larval development.

In each abdominal segment (one to seven) are found two ventrals, two spiraculars and four dorsal (two anterior and two posterior) histoblast nests. We imaged the dorsal posterior nest, which has the largest number of cells (15-17). We first imaged the adherens region, which plays an important role in epithelial morphogenesis ${ }^{22,23}$, with the fluorescent protein fusions E-cadherin:GFP or E-cadherin:mKate. We observed that histoblasts have straight cell borders and a polygonal shape up to about 90 hours after egg laying ( $90 \mathrm{~h}$ AEL). This morphology is the most commonly found in epithelia. In Fig. 1A-D we show adherens junctions of the same histoblast nest at different times between 90 and 115 hours AEL. After $90 \mathrm{~h}$ AEL, some cell junctions present local deformations (Fig. 1B), which become more prominent in the following hours (Fig. 1C). At the wandering stage, about $110 \mathrm{~h} \mathrm{AEL}$, the small wrinkles have become deep folds, with shapes that suggest mechanical buckling (Fig. 1D). These folds persist up to pupariation, when histoblasts initiate a series of fast cell cycle under the influence of accumulated cyclin $\mathrm{E}^{24}$. The emergence of the folds is well encapsulated by a quantification of cell circularity, which is the normalized ratio between the cell area and its perimeter (Fig. 1E). The circularity of a perfect circle is 1 , and it decreases as the overall shape is less round. Thus, cell circularity decreases as junctions fold. Fluctuating wrinkles of the adherens belt driven by the tension of the acto-myosin cortex often arise in different developmental contexts ${ }^{25}$. The typical lifetime of these fluctuations is of the order of minutes. By contrast, the folds we observe in the histoblasts are not dynamic, transient structures. The folds apparent in Fig. 1A-D evolve from wrinkles to fully developed lobes over a $20 \mathrm{~h}$ time window. No fluctuations of the cell junctions are observed in a period of minutes. Thus, with respect to the typical time scale of cytoskeletal fluctuations (seconds to minutes), junctional folds can be considered as quasi-static structures.

In addition to the circularity, we characterized histoblast shape transition through the quantification of their perimeter (Fig. 1F) and cell area (Fig. 1G). Interestingly, the cell perimeter slowly increases from 60 to $70 \mu \mathrm{m}$ between 90 and $100 \mathrm{~h}$ AEL, while it grows quickly up to $100 \mu \mathrm{m}$ after $100 \mathrm{~h}$ AEL. On the contrary, we observe a slow decrease of cell area between 90 and $100 \mathrm{~h} \mathrm{AEL}$, and faster decrease from $200 \mu \mathrm{m}^{2}$ at $100 \mathrm{~h}$ AEL to $120 \mu \mathrm{m}^{2}$ after $100 \mathrm{~h}$ AEL until the pupal stage. A decreasing area but an increasing perimeter results into a dramatic decrease of cell circularity, from 0.8 before the formation of folds, to 0.2 when the folds are fully formed.

To our knowledge, the only available data about epidermal growth in this system was obtained by electron microscopy and estimated from two dimensional data by ${ }^{19}$, who reported a 150 -fold increase in volume of LECs ans 60 -fold of histoblasts. While an increasing perimeter at the adherens plane seems compatible with growth of cells, we were surprised to observe a decrease in apical area. Hence, we investigated histoblasts growth in three dimensions, from the beginning of folds formation to the wandering stage (Fig. 2).

\section{As their apical surface shrinks, histoblasts grow basally}

We analyzed the 3D-shape of histoblasts in the course of the morphological transition by imaging their basolateral membrane with a src:GFP fusion and adherens junctions with an E-cad:mKate fusion (Fig. 2). Before the folds appear, cell borders have 


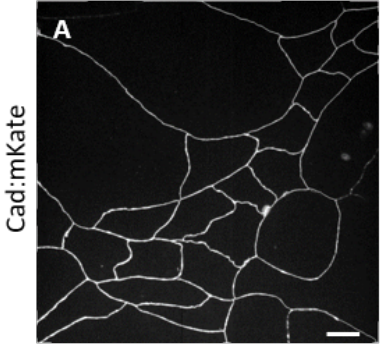

$90 \mathrm{~h}$ AEL

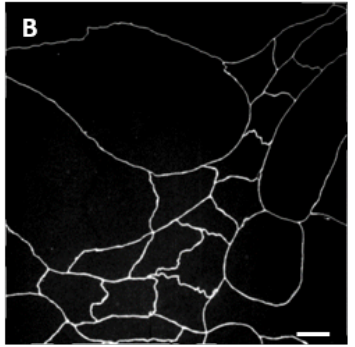

$95 \mathrm{~h}$ AEL

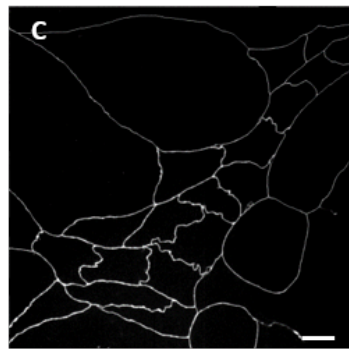

$98 \mathrm{~h} \mathrm{AEL}$

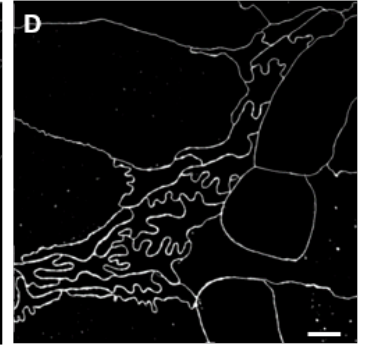

$115 \mathrm{~h} \mathrm{AEL}$
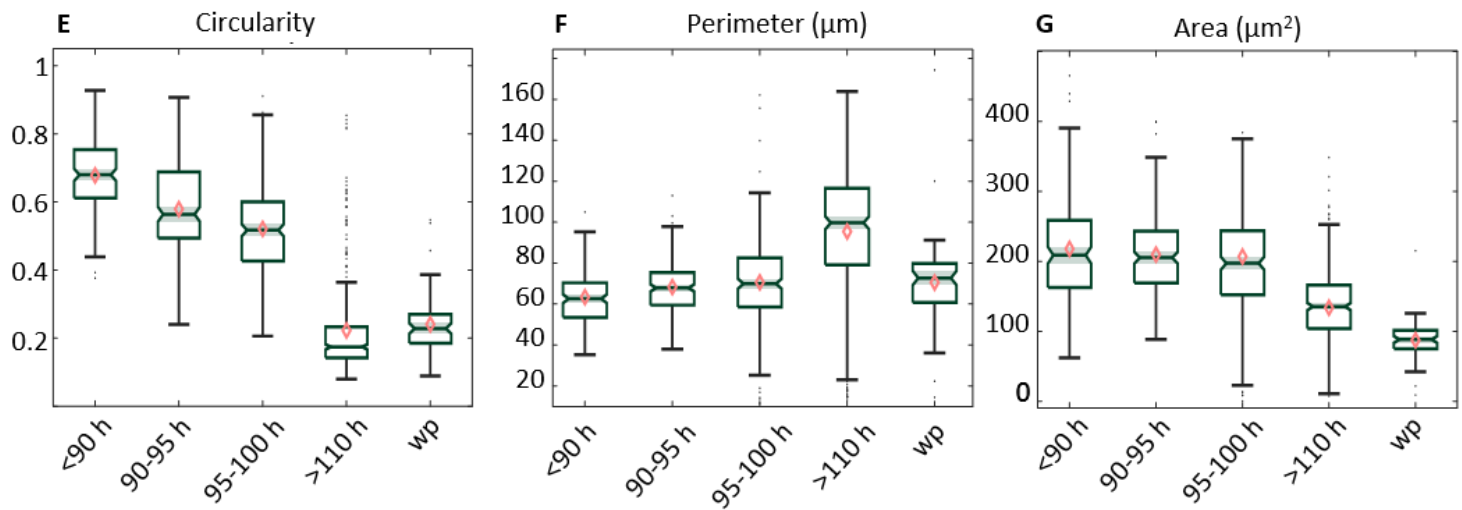

Figure 1. Remodeling of histoblasts' junction during epidermal morphogenesis. A-D: Chronic imaging of cadherin junctions at different times after egg laying (AEL). While at the beginning of the 3rd instar cell junctions are still straight, and develop more folds a the larvae grows, reaching a maximum before the pupariation, i.e. at the wandering stage. E-G:

Quantification of morphological parameters of histoblasts. Quantification of morphological parameters of histoblasts. Cell circularity ( $\mathbf{E})$ quantifies the shape change due to the formation of folds, with values ranging from 0 to 1 . A value of one corresponds to a perfect circle. Cell perimeter is growing as the larva grows $(\mathbf{F})$, while cell area decreases $(\mathbf{G})$. Scale bar $=10$ $\mu \mathrm{m}$. For each time interval, the number of analyzed cells is $\mathrm{N}=187, \mathrm{~N}=225, \mathrm{~N}=186, \mathrm{~N}=352, \mathrm{~N}=74$. 
the same straight shape at the adherens and basal planes, as can be seen in Fig. 2A-C. From the orthogonal projections in Fig. $2 \mathrm{C}(\mathrm{ZX}$ and $\mathrm{ZY})$ the apical and basal surface appear close to each other, with a total cell thickness of about $6 \mu \mathrm{m}$, which is comparable to the thickness of the LECs ${ }^{26}$. As junctions fold (Fig. 2D-F), the shape of histoblasts at the basal side assumes a different morphology than at the apical side. In particular, as shown in Fig. 2D, the nest is much larger basally than apically, and no junctional folding is visible basally. On the contrary, the basal side of histoblasts assumes a rounded, bulb-like shape. The difference in morphology of the apical and basal sides are striking on the overlay of the basal and apical membrane signal of Fig. 2G,H. Additionally, cell thickness is greatly increased in the course of the transition, with the apical and basal plane now being well separated, as shown from the orthogonal projections in Fig. 2F. The increase of cell thickness correlates well with the appearance of the folded phenotype, as demonstrated by plotting the distance between the basal and apical plane for different cell circularities (Fig. 2G). As reported in Fig. 2G, the average cell thickness increases from about $6 \mu \mathrm{m}$ to $10 \mu \mathrm{m}$ in the course of the morphological transition. As the apical area decreases while the height increases, we segmented histoblast nests after expression of a cytosolic GFP with a histoblast driver (esg-Gal4) to estimate their volume in the course of the transition. Because the cell number is constant in the histoblasts at this stage, the measurement gives us the cell-volume increase averaged over the nest. The measurements were done on individual nests, tracked over time through chronic imaging at three different time points. As reported in Fig. S2, cell volume increases 2-folds between 90 and 115 h AEL. Hence, histoblasts do grow during the last larval stage.

As shown in Fig. 2C, histoblasts are round-shaped at the basal side. Hence, the folded phenotype observed at the level of cadherin junctions is lost. To investigate whether the folds are only localized at the apical plane and lost immediately below, or whether they are gradually lost, we imaged septate junctions with a disc large protein fusion (dlg:GFP) together with E-cadherins (cad:mkate). Septate junctions are localized just below adherens junctions. Before junctional folds are formed, septate junctions have the same shape as adherens junctions. Their projections superpose (Fig. 2I). When folds appear, septate junctions follow only partially the shape of adherens junctions despite being localised very close to them (Fig. 2J). Thus cell border at the level of septate junctions are less folded than at the level of the adherens junctions but more than at the basal level.

In summary, over the time window from $90 \mathrm{~h}$ to $110 \mathrm{~h}$ after egg laying, histoblasts increase their volume two fold while their adherens junctional material also increase by a factor of 1.7. However, in that same time window, their apical area decreases. The volume increase is redistributed at the basal side of cells. We thus formulated a working hypothesis summarized in Fig. $2 \mathrm{H}$, according to which junctional folding in histoblasts would be generated by the competition for space of the two cell populations - histoblasts and larval epithelial cells- on a limited surface on the larval body. In this framework, compressive forces build up as the two cell populations grow on a limited surface. At some point, the growth of histoblasts does not proceed freely. The lateral constraints leads to shrinking of the apical surface of histoblasts, mechanical buckling of the growing junctions there, and volume redistribution at the basal side. The overcrowding effect could be enhanced by the slowing down of larval body growth after $90 \mathrm{~h} \mathrm{AEL}^{21}$ The junctions of histoblasts buckle, but those of LECs remain mostly straight. We explored whether some changes occur in the cytoskeleton of histoblasts that could affect the cortex in the adherens plane. Indeed, the critical load beyond which buckling proceeds depends in general on the stiffness of the compressed structure. The morphological transition could thus occur through a change in junctional stiffness.

\section{Junctional buckling is accompanied by a partial depletion of the cell cytoskeleton from the apical junctions}

The acto-myosin network plays a prominent role in setting the mechanical state of apical junctions ${ }^{22,23,27}$. Hence, we assessed the rearrangement of the acto-myosin network in the course of the folding transition. To do this, we first imaged flies carrying both cad:mKate and an affimer-GFP fusion to image actin (af:GFP, ${ }^{28}$, see methods) before and after the transition (Fig. 3). Actin colocalizes with cadherin both when junctions are straight (Fig. 3A,B) and after junctions have buckled (Fig. 3D,E). Nevertheless, after junctional folds have appeared, the actin signal is less neat, and a higher signal intensity is observed coming from the cell cytosol, at the apical plane (Fig. 3E). Additionally, both in $90 \mathrm{~h}$ and in $110 \mathrm{~h}$ larvae actin is also present at the basal side of the histoblasts (Fig. 3C,F) where the junctions are smooth and cells bulb-shaped as previously observed in src:GFP images (Fig. 2). Interestingly, from the orthogonal projections reported in Fig. 3C, F actin is visible at cell vertical junctions, connecting the apical and basal plane. This suggests that an actin relocation occurs reducing junctional actin and enriching basolateral junctions in the course of volume redistribution and cell-thickness increase. We quantified actin content at junctions by measuring the ratio between the junctional and cytosolic intensities of actin:GFP, at the same plane and for different circularity values (i.e. at different levels of junctional folding). The results of such quantification are reported in Fig. 3G. Details about the analysis and data treatment are given in the methods section and in Fig. S4. The quantification shows that junctional enrichment decreases with circularity, meaning that less actin is present at junctions when folds are forming.

We then imaged Myosin II through a GFP fusion of its regulatory light chain (Drosophila spaghetti squash, sqh:GFP) together with cad:mkte. As can be seen in Fig. 4, MyoII localizes at histoblasts junctions before the transition (Fig. 4A,B), but it is almost not visible anymore after buckled junctions have formed (Fig. 4C,D). Compared to E-cadherin (Fig. 4C), the Myosin II signal is more diffuse (Fig. 4D), and no enrichment at junctions is observed. In contrast to actin, no Myosin II is observed in 


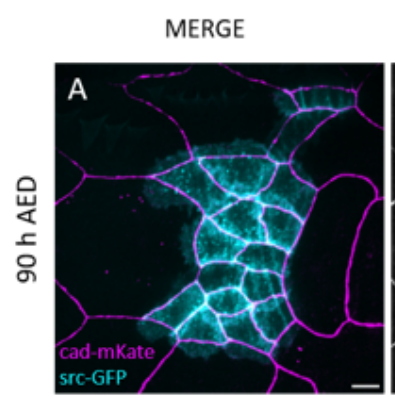

Apical - cell junctions

Basal - cell membrane
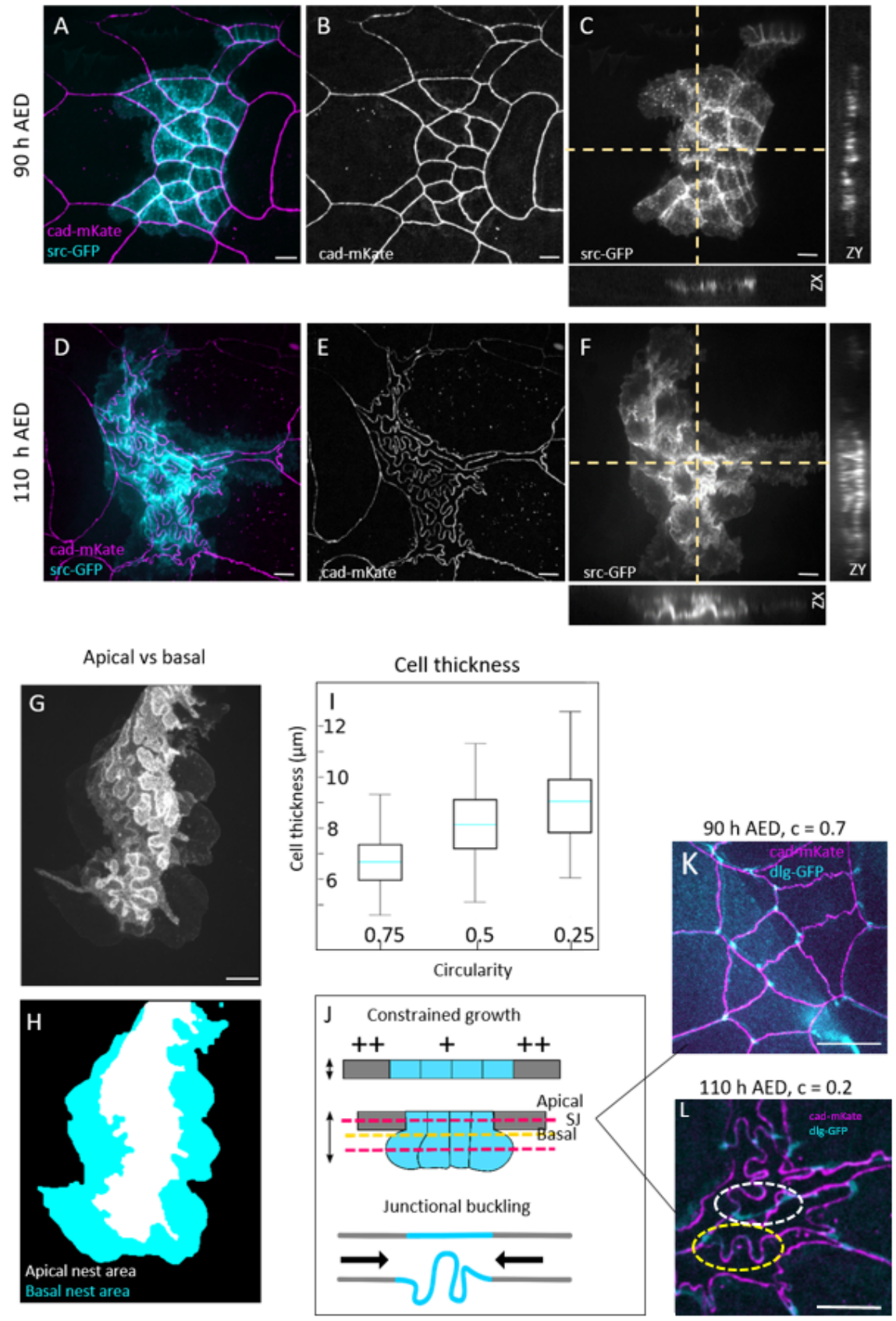

Figure 2. 3D characterization of histoblast growth and junctional buckling A-F: Live imaging of cadherin junctions (E-cad::mKate) and cell membrane (src::GFP) before (A-C) and after (D-F) apical junction remodeling. G,H: superposed projections of the apical and basal plane of a histoblast nest at $110 \mathrm{~h}$ AEL. While the apical side presents junctional folds, the basal side is characterized by large and bulb-shaped cells. I: Cell thickness plot as a function of cell circularity. Light blue line represent the means, error bars the standard deviation. J: Schematics of cell growth below the apical surface as junction remodeling occurs. At the top, section of histoblast and LECs showing how histoblast develop below the epithelial surface. Red dashed lines represent the apical and largest basal plane corresponding to images A-F. The yellow dashed line represents the plane of septate junctions, corresponding to images K and L. K,L: Merged projection of apical junctions and septate junctions at $90 \mathrm{~h}$ AEL (K), and $110 \mathrm{AEL}(\mathrm{L})$. Before junctional buckling, apical and septate junctions have similar, superimposing structures. When apical junctions fold, the deformation is partially lost at the plane of septate junctions. White dashed circle: example of straiht septate junction. Yellow dashed circle: example of septate junction not following the corresponding apical junction. Scale bar $=10 \mu \mathrm{m}$. 


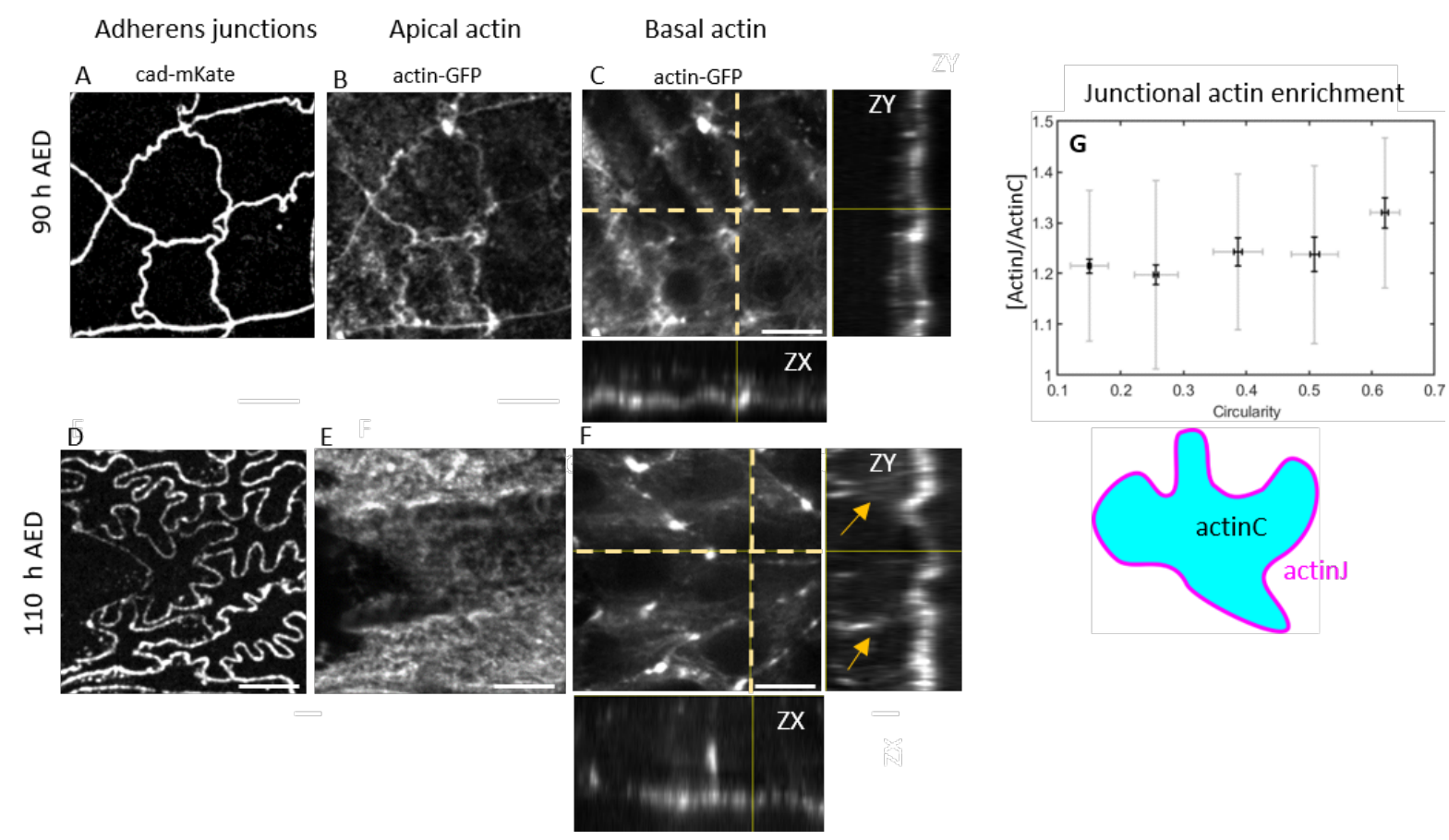

Figure 3. Localization of actin in histoblasts before and after apical junction remodeling. A-F: Live imaging of cadherin junctions and actin at $90 \mathrm{~h}$ AEL (A-C) and $110 \mathrm{~h}$ AEL (D-F). A,B: Projections of cadherin junctions and corresponding apical actin at $90 \mathrm{~h}$ and D, E: at $110 \mathrm{~h}$ AEL. C,F: Basal actin and orthogonal views at 90 and $110 \mathrm{~h}$ AEL, respectively, showing the xz and yz planes corresponding to the yellow dashed lines. The arrows in the zy plane show the actin structures joining the apical and basal planes. Scale bar $=10 \mu \mathrm{m}$. G: Plot of relative amount of junctional actin as a function of circularity, calculated as the ratio junctional signal/cystosolic signal at the same plane. The plot represents actin enrichment for each circularity bin as mean value +/- SD (gray) and SEM (black). actinJ = junctional actin, actinC = cortical actin, as represented in the schematic. A total of 23 histoblast nests with about 15 cells/nest were analyzed. The data were pooled and binned according to the circularity value.

the basal side of cells, where only a weak diffuse signal was visible (not shown). As previously done for actin enrichment, we quantified Myosin II content at apical junctions ad a function of cell circularity. As shown in Fig 4E, as junctions fold (i.e. as circularity decreases), Myosin II junctional enrichment decreases. Thus, junctional folding is accompanied by a gradual loss of Myosin II from cell junctions. The observed loss in Myosin II from junctions is likely to change the mechanical properties of histoblasts, possibly representing a reduction in stiffness which would facilitate buckling.

We could not identify the pathway that leads to Myosin-II depletion from junctions. In particular, the upstream regulator Rok does not seem to be implicated as no significant effect was observed on junction morphology when Rok activity was depleted (esgGal4 $>$ RokRNAi) or increased (esgGal4 $>$ RokCAT) in histoblasts, as reported in Fig. S3. Rok alterations in LECs (eip71>RokRNAi) did have a non autonomous effect on histoblast. Histoblasts still presented buckled junctions, but their overall shape appears less squeezed in the horizontal direction (Fig. S3D). Although sample variability was very high and the statistical analysis did not highlight any significant variation in Fig. S3D, the non-autonomous effect on histoblasts is in line with a mechanical tug of war between the two cell populations in the epidermis.

Overall, our data thus indicate that the change in mechanical properties of adherens junctions is linked with a change in the organization of the apical cytoskeleton of cells. Furthermore, the volume redistribution to the basal side is not an entirely passive mechanism as it is accompanied by the formation of vertical actin structures at the tri-cellular cell interfaces.

\section{The larval epidermis is not a tensed epithelium}

Our working hypothesis is that junctional folds are the results of lateral compression on the junctions of histoblasts. This implies that folding is not a tension-driven morphology. Additionally, we observe a loss of junctional myosin (Fig. 4), which also suggests a lack of tension in the tissue. To test this, we ablated both LECs and histoblast junctions during the morphological transition (around $95 \mathrm{~h} \mathrm{AEL).} \mathrm{We} \mathrm{performed} \mathrm{laser} \mathrm{ablation} \mathrm{on} \mathrm{cad:GFP} \mathrm{with} \mathrm{a} \mathrm{custom-built} \mathrm{setup} \mathrm{(see} \mathrm{Material} \mathrm{and} \mathrm{Methods).}$ First, we tested our system on a control, tensed epithelium - the adult epidermis after complete replacement of LECs by 

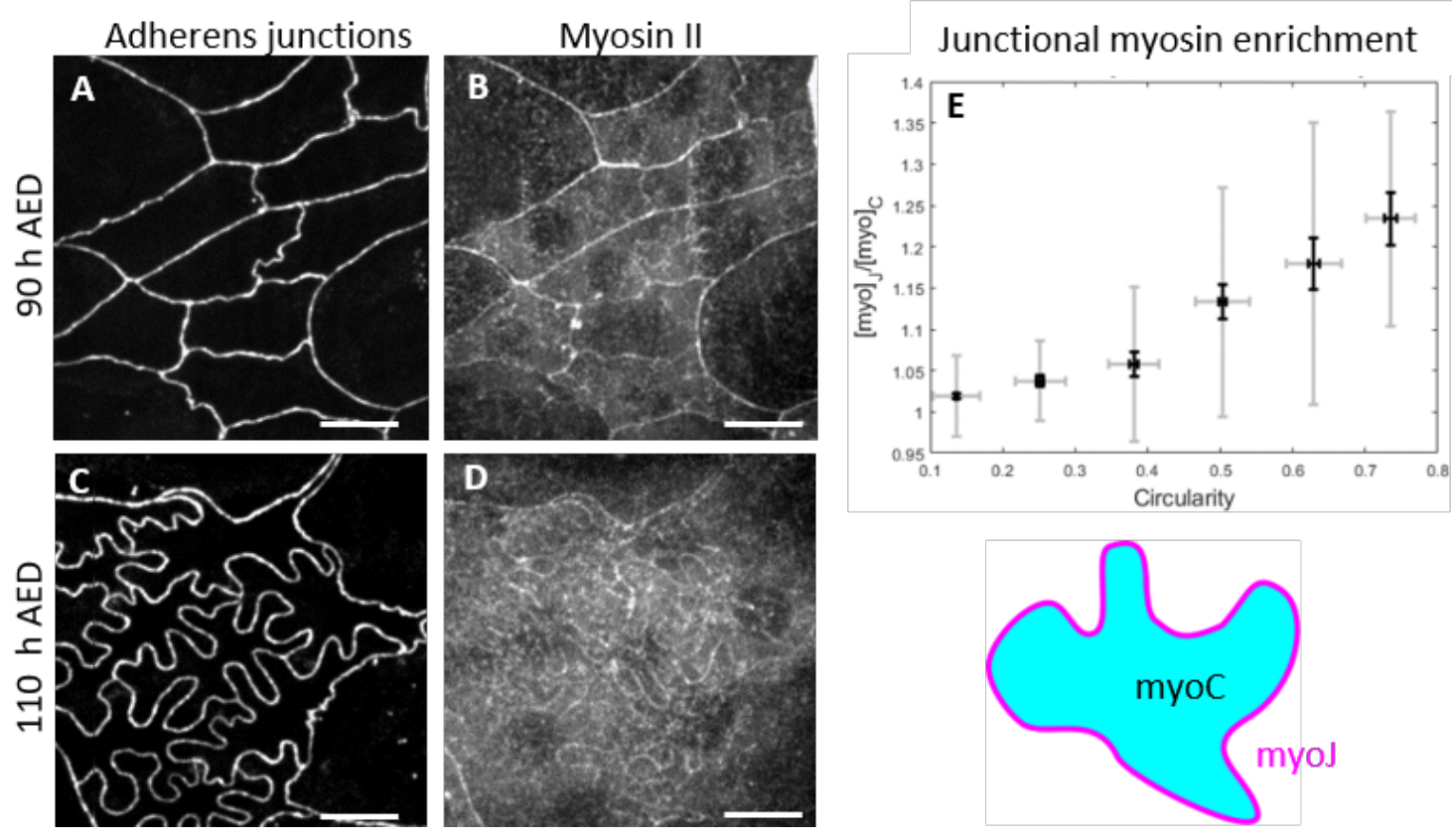

Figure 4. Localization of Myosin II in histoblasts before and after apical junction remodeling. Live imaging of cadherin junctions and corresponding apical myosin II at $90 \mathrm{~h}$ AEL $(\mathbf{A}, \mathbf{B})$ and $110 \mathrm{~h}$ AEL $(\mathbf{C , D})$.Scale bar $=10 \mu \mathrm{m}$. E Plot of relative amount of junctional myosin as a function of circularity, calculated as the ratio junctional signal/cystosolic signal at the same plane. The black and gray plot represents actin enrichment for each circularity bien as mean +/- SD (gray) and SEM (black). $\mathrm{MyoJ}=$ junctional myosin, $\mathrm{MyoC}=$ cortical myosin, as represeted in the schematic. A total of 29 histoblast nests with about 15 cells/nest were analyzed, and the data pooled and binned according to circularity values.

histoblasts in the pupal stage. When the adult epidermis was ablated, relaxation of the cut junctions was observed, as well as a shape change in the neighboring cells (Fig. 5A). Similar relaxations were observed in the wing imaginal discs, another tissue known to be under tension ${ }^{2,29-31}$. Instead, when either LECs or histoblasts were ablated at the larval stage, no relaxation was observed (Fig. 5 B,C). When we ablated histoblast junctions at the extremity of a lobe, no effect was observed (Fig. 5B and movie SM1). When LECs are ablated close to histoblast nest, no fast relaxation is observed in the course the first 80 seconds. A slow relaxation is observed over a few minutes, and no further relaxation during the next 30 min (Fig. 5B, movie SM1). After about $1 \mathrm{~h}$, the wound produced by laser ablation is repaired (Fig. $5 \mathrm{C}$ movie S2). These results confirm that epidermal morphology and homeostasis in 3rd instar larvae is not driven by a tension-based mechanism. These observations also demand a refinement of the buckling hypothesis. In fact, if the morphology of the junction were solely driven by buckling of an elastic body (the junction) under compressive forces, we should observe a straightening of the junctions upon ablation. Since this did not occur, our data suggest that some plastic remodeling must occur to stabilized buckled junctions and reduce the buildup of compressive forces.

\section{Junctional buckling in histoblasts is linked to cellular crowding of the epidermis}

In our hypothesis, as the two populations grow and compete for space on a limited surface, a lateral pressure builds up and constrains growth of histoblasts apically. We used genetic tools to validate the hypothesis that growth plays an essential role in junctional buckling. We impaired the insulin pathway specifically in LECs through the over-expression of a dominant-negative form of the Drosophila insulin receptor (dInR-DN) ${ }^{26,32}$. This resulted in a selective growth-reduction of the LECs. Figure $6 \mathrm{~A}$ shows a z-projection of a posterior nest surrounded by LECs in which growth was reduced. As compared to the wild type, histoblasts are characterized by a larger apical area (Fig. 6D), straighter junctions (Fig. 6F), but a perimeter of similar size as the wild type cells (Fig. 6E). Thus, less crowding through the reduction of the growth of LECs reduces junctional buckling of histoblasts in a non-autonomous way. This result supports the hypothesis that the laterally pushing LECs are at the origin of both the formation of buckled junctions and the reduction of histoblast apical area.

In our hypothesis, histoblast buckling is a form of remodeling driven by the concomitant action of the lateral compression from LECs and the growth of the histoblast junctions themselves. We thus specifically altered junctional growth in histoblasts by genetic means, impairing the activity of Rab11, known for its role in cadherin recycling. In particular, Rab11 is responsible for the transportation of newly-synthesized cadherin as well as recycled caherin and other proteins to the cell junctions $\left({ }^{33}\right)$. We 


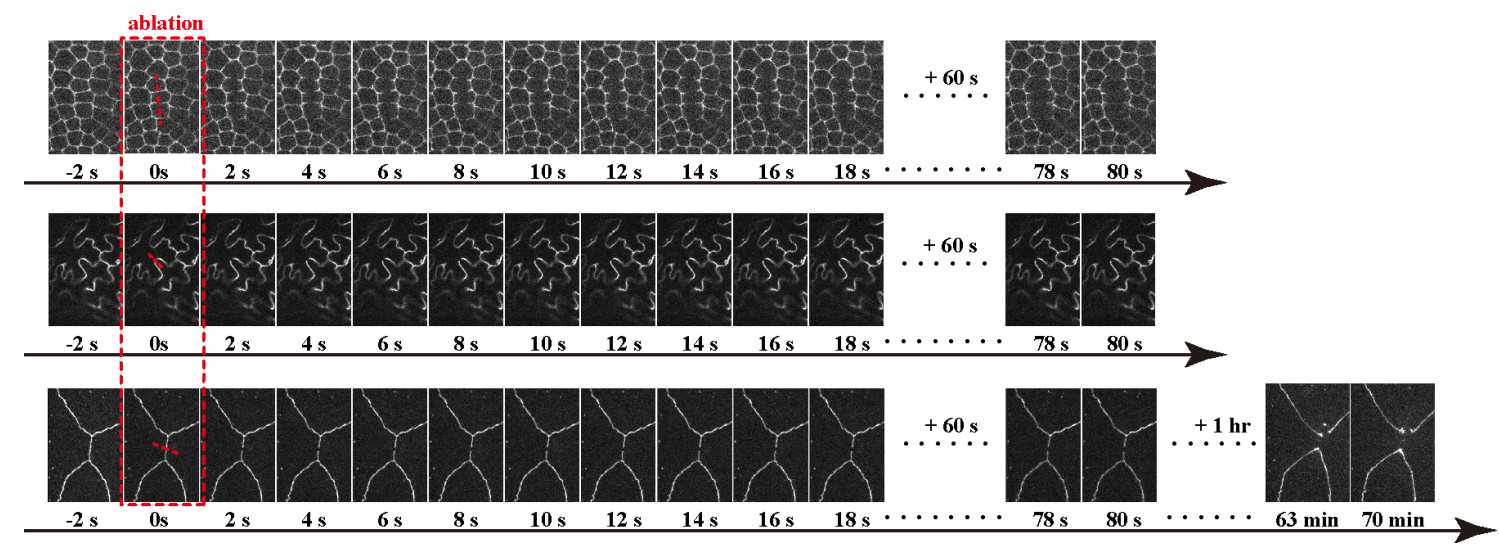

Figure 5. Laser ablation of larval epithelium. A Laser ablation of pupal epidermis. B: Laser ablation of histoblasts in a $95 \mathrm{~h}$ AEL larva. C: Laser ablation of LECs in a $95 \mathrm{~h}$ AEL larva, close to histoblasts.

induced the over-expression of a Rab11 dominant negative (Rab11-DN) specifically in the histoblasts. As shown in Fig. 6B, the effect is that histoblasts have shorter junctions (40 vs $110 \mu \mathrm{m}$ as in the wild type), smaller apical area (50 vs $150 \mu \mathrm{m}^{2}$ ) and do not undergo junctional buckling.

Combined, these perturbation experiments provide strong evidence that junctional buckling of the histoblasts is the result of a imbalance between the addition of junctional material in the histoblasts and mechanical constraints from the overcrowding of the epidermis.

\section{Reintroduction of cell division abolishes junctional folding}

Unlike imaginal discs, the other precursors of adult tissues in the Drosophila, histoblasts do not divide while they grow. As a consequence, cell-cell interfaces of the histoblasts lengthen in the course of the larval stages (Fig. 1). The length of junctions may be an important intrinsic factor that control the buckling transition. Indeed the critical compressive load at which buckling proceeds for an elastic beam scales as $\sim L^{-2}$, where $L$ is the characteristic length of the beam. Besides the alteration of Rab11 function, an alternative way to alter the size distribution of junctions is to reintroduce cell divisions in histoblasts.

We forced histoblasts to divide by over-expressing the mitotic controller cdc25 (string in Drosophila) ${ }^{34}$. This perturbation only impacts the cell cycle and not growth ${ }^{35}$. As a consequence, histoblasts were more numerous in the histoblasts nests (around 100). As shown in Fig. 6C,F, individual histoblasts are consequently smaller and have straight junctions. This experiments confirms that junctional buckling is a result of the combined overcrowding and absence of divisions.

Besides the direct effect on cell junctions, the whole histoblast nest is also larger than in the control Fig. 6C. A possible interpretation for this experiment is that the shortened length of junctions prevents the onset of buckling and thus improves their ability to withstand mechanical stress. As the junctions are less prone to buckling, the overall nest becomes stiffer and gets squeezed to a lesser degree. Thus, forcing cell divisions in histoblasts abolishes buckling and reduces compaction of the histoblast nest.

\section{Qualitative model of junctional buckling}

Based on our results, we formulated a descriptive model that we called the junctional buckling model (Fig. 7). According to our model, LECs and histoblasts form a continuous cell layer which is initially homogeneously thin (few $\mu \mathrm{m}$ ), with LECs and histoblasts sharing similar polygonal morphologies, but different sizes. None of the populations divides, but both grow. At the end of the larval phase, cellular crowding of the epidermal surface generates a mechanical constraint on the entire histoblast nest. A loss of junctional myosin changes the mechanical properties of histoblasts' junctions, which become less stiff, but are still increasing in length. The increased length of histoblast-junction reduced stiffness, and compressive load from cellular crowding altogether induce buckling of histoblast junctions, with three main consequences on the epithelial morphology: I) Histoblasts junctions lobulate, II) their apical area squeezes, and III) the cell volume is redistributed below the apical surface. Such a volume redistribution is necessary to accommodate the increased mass of growing cells, while the apical surface is reduced.

The junctional buckling contrasts with conventional buckling in several ways. First, junctional buckling proceeds at shorter spatial length than the total length of the junction, the latter being expected when a simple elastic beam experiences compressive load. High frequency modes are expected when the compressed structure is coupled to an elastic structure. They have been observed for example on buckled microtubules in contractile cells ${ }^{36}$. Second, the precise shape of histoblast-junctions seem to 


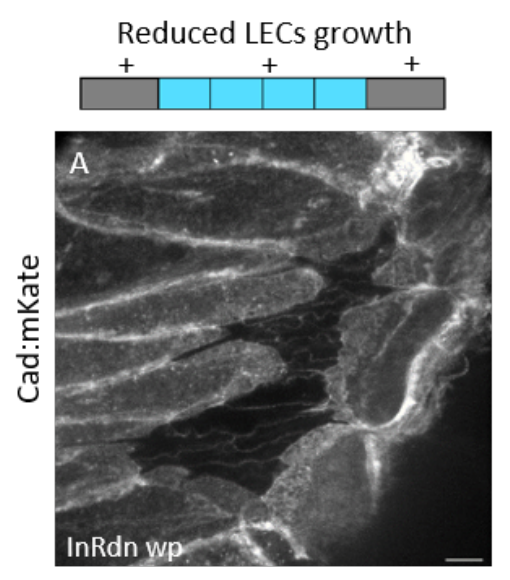

Impaired hb junction growth
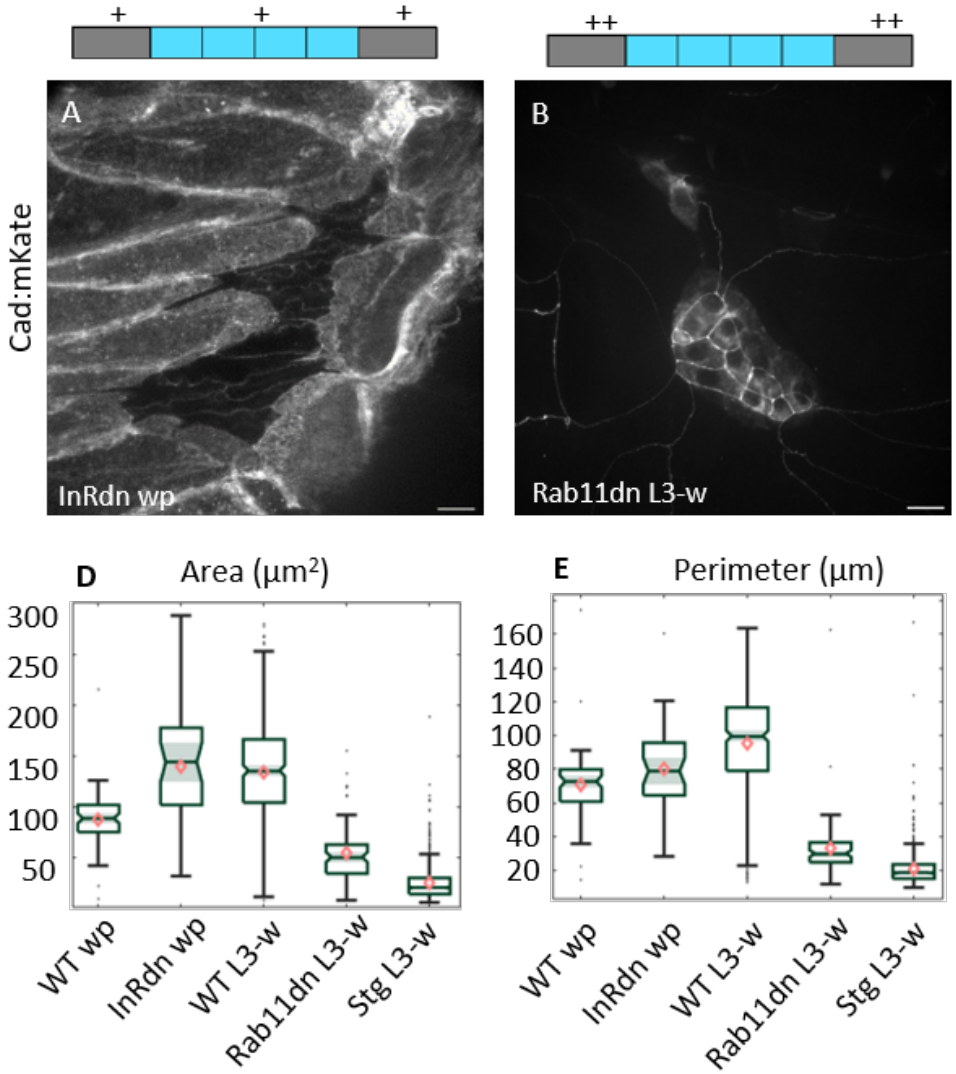
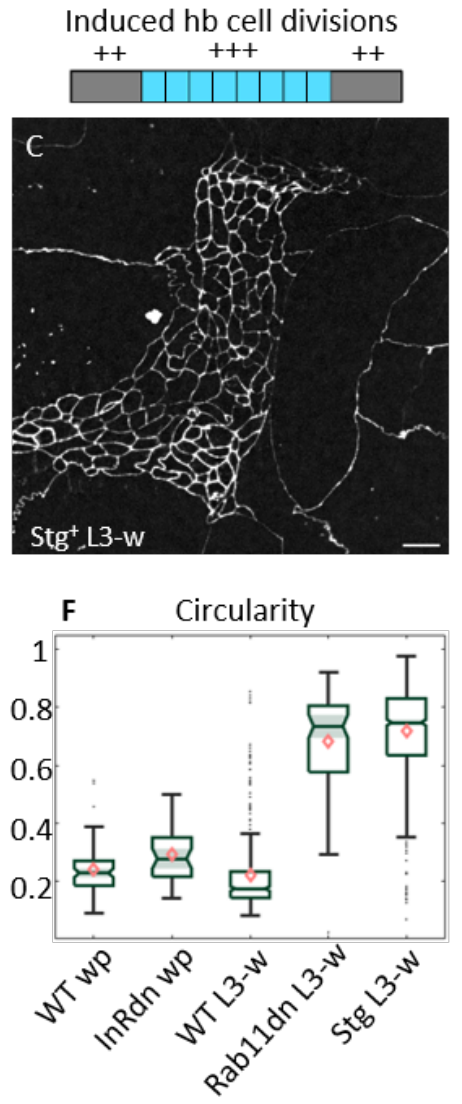

Figure 6. Genetic perturbation of larval epidermis . A: Drosophila epidermis (end of larval stage here, also called white pre-pupa, wp) when LECs growth was reduced by impairing the insulin receptor pathway (cad::mkate; e22c-Gal4 > UAS-InRdn UAS-GFP). A cytosolic GFP was co-expressed with InRdn to confirm that gene expression is confined to LECs. A reduced junctional buckling is observed in histoblasts. B: Live image of a wandering stage larva in which histoblast junction recycling was impaired by a dominant negative form of Rab11 (cad::mkate; esg-Gal4 > UAS-Rab11dn). Rab11dn histoblasts show shorter, straight junctions and reduced apical area. C: Epidermis of a wandering stage larva in which cell divisions in histoblasts was forced by overexpressing cdc25/stg (cad::mkate; esg-Gal4 > UAS-Stg). Histoblast are more numerous, smaller and buckling is abolished. D-F: Quantification of the essential descriptors: cell area (D), perimeter (E), circularity (F). Scale bar $=10 \mu \mathrm{m}$. WT = wild type, wp = white pre-pupa, L3-w = wandering stage larva. $\mathrm{N}$ cells $=352(\mathrm{wt}), 38(\mathrm{InRdn}), 82$ (Rab11dn), 880 (Stg+) 


\section{JUNCTIONAL BUCKLING MODEL}

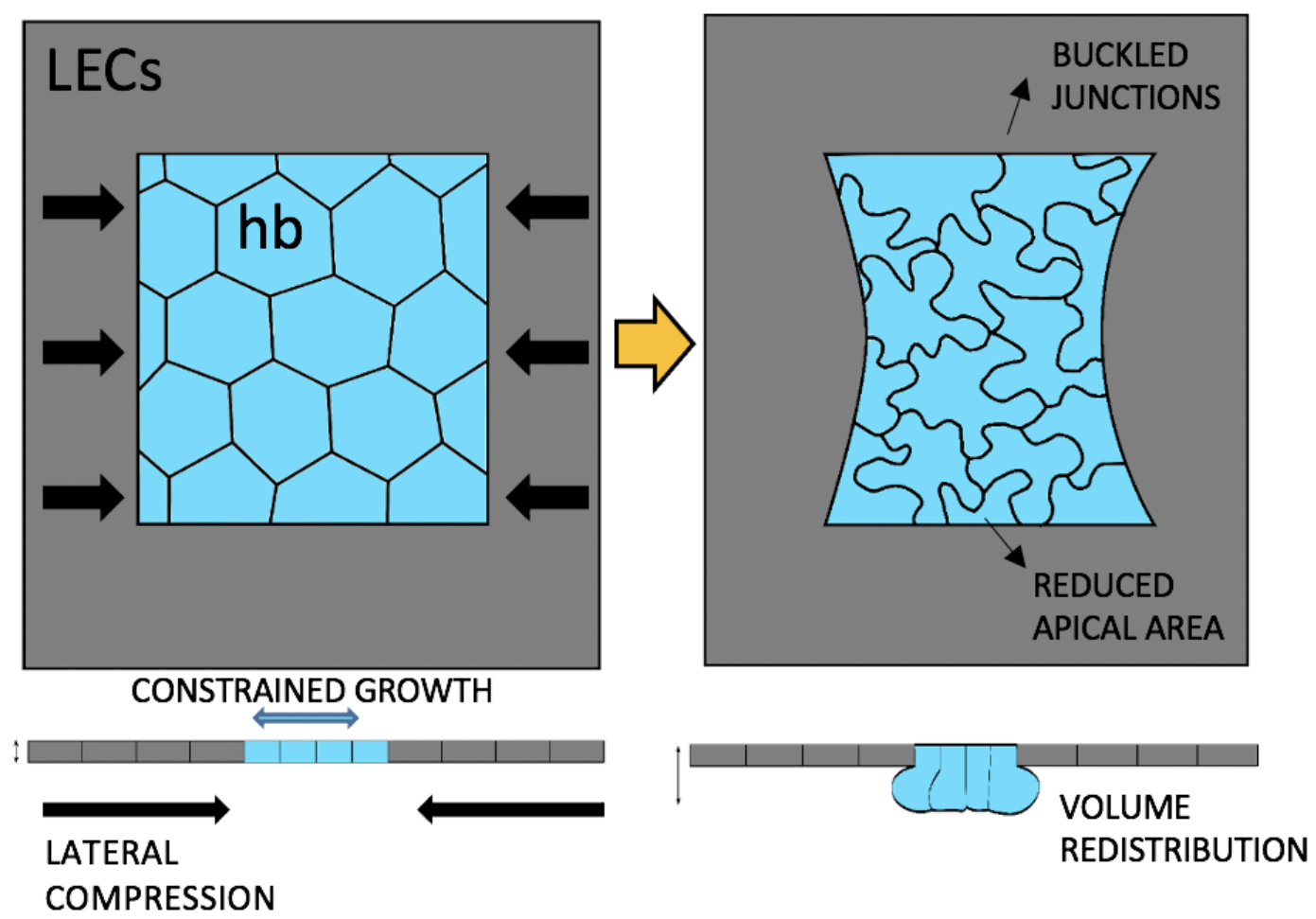

Figure 7. Junctional buckling model Qualitative model of histoblasts' morphological transition. Histoblast, in blue, are surrounded by LECs, which grow at a faster rate, hence applying a lateral force to histoblast at the apical plane. While histoblast are also growing, their growth is constrained. This competition for the available space results in three main consequences on the 3D geometrical configuration of the weak population (histoblast): cell junctions buckle under the lateral push, histoblast apical area decreases, and histoblast grow below the apical surface, hence redistributing their cell volume. 
be stabilized by a plastic process, as evidenced by the fact that rupture of the junctions does not straighten the junction (Fig. 5).

In our model, the two histoblast-intrinsic factors that contribute to buckling are the absence of cell divisions in the growing histoblasts, which makes adherens junctions lengthen continuously, and the softening of the junctions through a loss of cytoskeletal components. These two factors are poised in a such a way that the apical junctions buckle as crowding increases, which effectively compacts histoblasts.

\section{Discussion}

We have presented a novel case of epithelial morphogenesis in which most of the canonical rules for epithelial development are no longer valid. In recent years, an emphasis has been put on the importance of junctional tension in shaping epithelial cells $^{2,37,38}$, and the release of mechanical stress through topological transitions ${ }^{6,7,39}$. Instead, the larval epidermis of Drosophila is formed by two cell populations which are growing but not dividing and not exchanging neighbors. We observe that histoblasts' junctions evolve into a deeply folded phenotype (Fig. 1), very different from the tensed straight cell interfaces usually found in epithelia. Such unusual phenotype, which we named junctional buckling, is stable over several hours.

We investigated the formation of histoblast junctional folding and found that it is a non-autonomous transition originated by the competition for space of the two cell populations. We found that buckling correlates with a relocation of the cytoskeletal proteins actin and myosin II from histoblasts' junctions (Fig. 3, 4). This cytoskeletal depletion of junctions may help their folding by reducing their stiffness, owing to the fact that the critical buckling load of a beam scales linearly with the stiffness. Thus folding may proceed at the onset of a buckling instability induced both by an increased lateral compression and a reduced stiffness of the junctions and greater propensity to bend. To support our idea of a compressive force due to an overcrowding, we altered the mechanical equilibrium by genetically modifying either growth or cell divisions. Limiting the growth of LECs or the lengthening of histoblast junctions results in the reduction, and in some cases complete abolition, of junctional buckling (Fig. 6 A,B). Notably, the non-autonomous effect of reduced growth of LECs on buckling of histoblast junctions is a strong indicator that junctional buckling of histoblast is not driven exclusively by a cell autonomous program but also results from the mechanical tug of war between the two populations. Similarly, when we reintroduced cell divisions (Fig. 6C), the number of junctions was multiplied and histoblasts provided with a resisting force against the pushing LECs. Hence, we found the typical morphology of straight cell junctions. Because no effect is observed when folded junctions are ablated, we speculate that a plastic remodeling of junctions takes place that dissipates the stress due to lateral compression. Hence, when tissues are not under tensions, growth-related stresses lead to stable phenotypes which do not belong to the known phase space of tensed epithelia ${ }^{2}$.

The buckled junctions are reminiscent of fluctuating junctions as observed both in Drosophila and in other organisms. For example, during early dorsal closure, amnioserosa cells show wrinkled cell junctions due to fast contractions of the acto-myosin cytoskeleton $^{25,40-42}$. A closer look however indicates that these are very different mechanisms : the typical time scale of these fast contractions is of the order of seconds. Instead, junctional folds in histoblasts gradually fold over a period of hours and last up to the pupal phase, when cell divisions start. A closer cellular process is at play in the pavement cells of plant leaves ${ }^{43,44}$. These "puzzle-shaped" cells are very similar to histoblasts in that they also develop over a long time interval and stabilize by a plastic remodeling ${ }^{43}$. Although the final shape of plant pavement cells closely resembles that of histoblasts, their makeup seems to operate in reverse. Plants have a rigid cell wall that is under tension from turgor pressure. In the case of the puzzle shape of pavement cells, the formation of lobes is driven by growth restriction in a field under tension. Instead, the formation of lobes in histoblasts is due to lateral compression, which we believe is then compensated for by a plastic remodeling. With physical mechanism operating in reverse, compression vs tension, histoblasts and pavement cells are examples of stable phenotypes that differ from the usual polygonal shape, and that are governed by other mechanisms than acto-myosin tension.

Is there an advantage for histoblasts to go through junctional buckling? We propose that this folding of junctions allows the entire histoblast nest to remain compact while its constitutive cells are growing in a crowded epidermis. Owing to the potential effects of compressive forces on cell physiology (reviewed $\mathrm{in}^{45}$ ), this compaction of the nest may serve to avoid the crowding to reach deleterious levels. The compact, folded state is made possible by the absence of cell divisions (Fig. 6C) and the softening of the junctional cortex (Fig. 3,4). Through this mechanism, the delay of cell divisions to the pupal stage (a stage when the larval epidermis will degrade) may thus serve to protect histoblast by taming the compressive stress. Alternatively, just keeping the growing nest as small as possible reduces the chance that it will be damaged accidentally in the larval life. It is interesting to compare the growth of histoblasts with that of imaginal discs, which are the other precursors of adult tissues in Drosophila. Imaginal discs grow as isolated luminal epithelia attached to the larval carcase through only a thin stalk. As opposed to histoblasts, imaginal cells do divide as they grow ${ }^{46}$. This may be linked to the fact that they do not experience the overcrowding like histoblasts, as imaginal discs are not contiguous to any other growing tissue.

To conclude, crowding of the Drosophila larval epidermis compresses growing junctions of histoblasts, giving rise to changes in shape linked with buckling. Histoblasts are poised in a mechanical regime yet unexplored to our knowledge. In 
future work, it will be interesting to address how junctional buckling differs from classical buckling of elastic beams - in particular to understand the nature of the plastic process which stabilizes the folds as they form.

\section{Methods and Materials}

\section{Fly stocks}

D. melanogaster strains were grown at $25^{\circ} \mathrm{C}$ except if else stated, in standard food (Nutri-Fly "German formulation"). A list of all the strains used for this study and generated for this study is listed in Table 1. For snapshot-imaging of larvae at the same stage, eggs were collected every 4 hours from the culture tube. The age is thus expressed in hours after egg laying (h AEL), and the results averaged of a time window of 4 hours.

\begin{tabular}{|c|c|c|}
\hline Stock description & Short name & Origin \\
\hline \hline ;endo-Ecad:mKate(2x)/ CyoGFP; & cad:mKate & Y. Bellaiche \\
\hline ; endo-cad:GFP; hist:RFP & cad:GFP & C. Collinet \\
\hline ; esg-Gal4 endo-cad:GFP ; & esg-Gal4 cad:GFP & this study \\
\hline ; esg-Gal4 endo-cad:GFP ; Gal80ts /TM6b & esg-Gal4 cad:GFP Gal80ts & this study \\
\hline esg-Gal4 endo-cad:mKate & esg-Gal4 cad:mKate & this study \\
\hline ; Eip71CD-Gal4 ; & Eip71-Gal4 & Bloom. 6871 \\
\hline ; Eip71CD-Gal4, cad::mKate & eip71-Gal4 cad:mKate & this study \\
\hline ;UAS-Src:GFP; & UAS-src:GFP & P. Kakanj \\
\hline yw;:UAS-InR(DN) & UAS-InR(DN) & Bloom. 8253 \\
\hline UAS-stg.N/CyO & UAS-stg & Bloom. 4777 \\
\hline w[*] dlg1[YC0005] & Dlg:GFP & Bloom. 59417 \\
\hline sqhAx3; sqh-sqh:GFP; sqh-sqh:GFP & UAS-Rab11dn & Bloom. 66675 \\
\hline w;; UAS-Rab5-S43N & UAS-Rab5DN & J. Solon \\
\hline w[*]; shi[ts] & UAS-Shi[ts] & Bloom.66675 \\
\hline y[1] w[*]; UAS-Rok.CAT & UAS-RokCAT & Bloom.6668 \\
\hline y[1] v[1]; UAS-RokRNAi & UAS-RokRNAi & Bloom. 28797 \\
\hline ; UASp-affimer06:GFP & UAS-aff06:GFP & M. Mavrakis \\
\hline
\end{tabular}

Table 1. stocks. Bloom. = Bloomington stock center. Short name $=$ name used in the main text.

\section{Live imaging}

Live imaging was performed with a custom built confocal spinning disc setup built of an inverted microscope (EclipseTi2-E, Nikon Instruments), and a spinning disc device (CSU-X1-M1, Yokogawa). Images were acquired with a 488 and a $561 \mathrm{~nm}$ lasers (Sapphire, Coherent) and an iXon Ultra888 EMCCD camera (Andor,Oxford Instruments). Z-stacks were acquired with a z-interval of 1 . Laser power and exposure were kept as low as possible for chronic imaging, to reduce phototoxicity. The two color channels (GFP and mCherry, 488 and $564 \mathrm{~nm}$ lasers, respectively), where acquired in sequence. All images were obtained with a 60X water-immersion objective (Plan Apo 60×, NA 1.2, Nikon).

\section{Snapshot imaging}

We refer to snapshot imaging when each larva was imaged just once, at a specific time. To characterize the buckling transition, we imaged staged cad:mCherry larvae at different ages (hours AEL). To compare different mutants and obtain other measurements (i.e. myosin and actin content, cell volume, cell thickness), we chose larvae at the wandering stage, when the buckling transition has accomplished. Larvae were anesthetized with a custom-built chamber made with a glass-bottom Petri dish (MakTek), and a 3D-printed lid with two inlets (Fig. S6), connected to two syringes via rubber tubings (VWR, Tygon 3603). One tube can be closed by by a two-way manual valve (Masterflex, 30600-00) and the other by a three-way valve (Masterflex, 30600-01 ). $200 \mu l$ of Desflurane (Suprane, Baxter) were injected in one syringe, then the syringe closed to $5 \mu 1$ and the liquid let expand to about $18 \mu$ l, by closing the valve. In the meanwhile, larvae were washed in PBS and placed in the Petri dish. After a first anesthetization of about 5 minutes, the valves were closed, and the Petri dish open to allow alignment of the larvae to image histoblasts. The anesthetic was then reinjected and the larvae imaged immediately after. 


\section{Chronic imaging}

We refer to chronic imaging when the same larvae were imaged several times, at different ages. The anesthetization and imaging protocol adapted from ${ }^{47}$ is schematized in S1. Larvae were washed and anesthetized as described above, but with a lower anesthetic dose $(150 \mu \mathrm{l})$. To avoid potential effects on growth due to starvation, we limited the anesthetization time (including imaging) to about 30 minutes. After each imaging session, larvae were put one by one in a humid chamber with soft food and incubated at $25^{\circ} \mathrm{C}$. With this protocol, all analyzed larvae survived to the adult phase.

\section{Laser ablation experiments}

Laser-ablation experiments in were implemented on a home-built system described in Meng et al. ${ }^{48}$. The system couples a near infrared 130 fs mode-locked Ti: sapphire laser (YLMO 930 $\pm 10 \mathrm{~nm}$, MenloSystems) operating at $130 \mathrm{MHz}$ to an inverted Nikon Eclipse Ti microscope (Nikon Instruments) equipped with a Yokogawa spinning disk unit (CSU-X1, Yokogawa Electric) for performing ablation and recording of sample perturbation after ablation.

\section{Laser ablation}

Ablation of epithelial junctions were performed by tightly focused the NIR laser in the focal plane using a water-immersion objective (Plan Apo 60×, NA 1.2, Nikon) and realised plasma ablation. To generate line ablations the laser beam was moved 2 to 3 times along target region in the sample with the help of a Galvano scanner (Cambridge Technologies) at a constant speed of about $500 \mathrm{ums}^{-} 1$ with an average power of $154 \mathrm{~mW}$ at the back aperture of the objective.

\section{Time-lapse imaging}

Acquisition was performed before and after ablation with the help of $488 \mathrm{~nm}$ diode laser ( $2 \mathrm{~mW}$ nominal, coherent OBIS LX) by using the same microscope as ablation. For all ablation experiments, an initial GFP frame was acquired prior to laser ablation and located the ablation region. For larval epidermal cells (LECs) and histoblasts, time-lapse imaging was acquired at a frame rate of $5 \mathrm{fps}$ to visualize the ablation process and the changes of ablation region.

\section{Image analysis}

Image processing and data analysis were mainly performed in Matlab using custom-written scripts. Preprocessing Image segmentation of cad:mKate and cad:GFP projections were done in Ilastik ${ }^{49}$ and TissueAnalyzer for ImageJ.

\section{Morphological analysis of histoblasts' junctions}

For morphological analysis, z-stacks of cadherin junctions obtained by confocal spinning disk microscopy were projected by simple maximum projections, when the tissue was well positioned and parallel to the glass slide, or by a curved projection when the histoblast plane was tilted relatively to the imaging plane. The surface detection algorithm and curved projection were performed using the procedures described in Abouakil et al. ${ }^{50}$. The maximum intensity projections were then segmented using Ilastik ${ }^{49}$ and Tissue analyzer ${ }^{51}$. The segmentations were then used to calculate cell area, perimeter and circularity. We represented each parameter and group of data as a box plot containing the mean value (diamond-shape marker), median (middle solid line), lower and upper quartiles (box limits), outliers (dots), and minimum and maximum non-outliers values. The notches and shaded regions represent the $5 \%$ confidence interval, i.e. if two boxplots have superposed notches, the two data set are considered from the same Gaussian distribution.

\section{Myosin and actin content analysis}

The surface-detection algorithm by Abouakil et al. ${ }^{50}$ was used to define the plane of cadherin junctions. The identified surface was then used as a mask to analyze the fluorescence signal coming from junctional actin or myosin. The junctional enrichment was then calculated as the ratio of the normalized intensity of the junctions and of the cytosol, at the corresponding plane.

\section{Volume estimation}

cad:mkate esg $>$ GFP flies were imaged to estimate the total nest volume, as a function of the average cell circularity. We analyzed Cad:mKate stacks as described in the morphometric analysis section. To obtain an estimate of the volume, we first equalized the intensity values of all stacks, then used Ilastik to obtain 3D-segmentation of the GFP signal. Finally, we analyzed and plotted the data with Matlab, MathWorks.

\section{Thickness measurements}

Histoblast thickness was measured from cad:mkate esg $>$ src:GFP flies. Cad:mKate stacks were analyzed as described above. Esg > src:GFP stacks were analyzed using a custom script coded in Matlab as follows. For each xy pixel, the z signal was fitted to a double Gaussian to identify the positions of the intensity peaks, i.e. of the apical and basal membrane at each position. For each nest we thus calculated the average thickness as the average distance between the two membranes. Data from different stacks were then pooled to obtain average values as a function of cell circularity. 


\section{Acknowledgments}

We thank Manos Mavrakis, Sophie Brasselet, Raphael Clément, Martine Ben Amar, Richard Smith, Adam Runion for fruitful discussions on this project. We thank Frédéric Galland for advice on image analysis. We thank Yohans Bellaiche, Jérome Solon, Manos Mavrakis, Claudio Collinet and Parisa Kakanj for sharing stocks.

This work was funded by the following agencies: Agence Nationale de la Recherche (ANR-18-CE13-028, ANR-17-CE30-0007) ; Excellence Initiative of Aix-Marseille University - A*Midex (capostromex), a French Investissements d'Avenir programme; The project leading to this publication has received funding from the « Programme d'Investissements d'Avenir $\gg$ of the French Government, managed by the French National Research Agency (ANR-16-CONV-0001, ANR21-ESRE-0002), and from Excellence Initiative of Aix-Marseille University - A*MIDEX.

\section{References}

1. Gibson, M. C., Patel, A. B., Nagpal, R. \& Perrimon, N. The emergence of geometric order in proliferating metazoan epithelia. Nature 442, 1038-1041 (2006).

2. Farhadifar, R., Röper, J. C., Aigouy, B., Eaton, S. \& Jülicher, F. The Influence of Cell Mechanics, Cell-Cell Interactions, and Proliferation on Epithelial Packing. Curr. Biol. 17, 2095-2104, 10.1016/j.cub.2007.11.049 (2007).

3. Lim, C. T., Zhou, E. H. \& Quek, S. T. Mechanical models for living cells-a review. J. biomechanics 39, 195-216, 10.1016/j.jbiomech.2004.12.008 (2006).

4. Cai, Y. \& Sheetz, M. P. Force propagation across cells: mechanical coherence of dynamic cytoskeletons. Curr. opinion cell biology 21, 47-50, 10.1016/j.ceb.2009.01.020 (2009).

5. Heisenberg, C. P. \& Bellaïche, Y. XForces in tissue morphogenesis and patterning. Cell 153, 948, 10.1016/j.cell.2013.05.008 (2013).

6. LeGoff, L. \& Lecuit, T. Mechanical forces and growth in animal tissues. Cold Spring Harb. Perspectives Biol. 8, 1-18, 10.1101/cshperspect.a019232 (2016).

7. Baum, B. \& Georgiou, M. Dynamics of adherens junctions in epithelial establishment, maintenance, and remodeling. $J$. Cell Biol. 192, 907-917, 10.1083/jcb.201009141 (2011).

8. Takeichi, M. Dynamic contacts: Rearranging adherens junctions to drive epithelial remodelling. Nat. Rev. Mol. Cell Biol. 15, 397-410, 10.1038/nrm3802 (2014).

9. Foolen, J., Yamashita, T. \& Kollmannsberger, P. Shaping tissues by balancing active forces and geometric constraints. Curr. Opin. Cell Biol. 49, 053001, 10.1088/0022-3727/49/5/053001 (2015).

10. Ambrosi, D. et al. Growth and remodelling of living tissues: perspectives, challenges and opportunities. J. The Royal Soc. Interface 16, 20190233, 10.1098/rsif.2019.0233 (2019).

11. Lee, T., Holland, M. A., Weickenmeier, J., Gosain, A. K. \& Tepole, A. B. The geometry of incompatibility in growing soft tissues: Theory and numerical characterization. J. Mech. Phys. Solids 146, 104177, 10.1016/j.jmps.2020.104177 (2021).

12. van Leen, E. V., di Pietro, F. \& Bellaïche, Y. Oriented cell divisions in epithelia: from force generation to force anisotropy by tension, shape and vertices. Curr. Opin. Cell Biol. 62, 9-16, 10.1016/j.ceb.2019.07.013 (2020).

13. Alt, S., Ganguly, P. \& Salbreux, G. Vertex models: From cell mechanics to tissue morphogenesis. Philos. Transactions Royal Soc. B: Biol. Sci. 372, 10.1098/rstb.2015.0520 (2017).

14. Fletcher, A. G., Cooper, F. \& Baker, R. E. Mechanocellular models of epithelial morphogenesis. Philos. Transactions Royal Soc. B: Biol. Sci. 372, 20150519 (2017).

15. Gibson, W. T. \& Gibson, M. C. Cell topology, geometry, and morphogenesis in proliferating epithelia. Curr. topics developmental biology 89, 87-114 (2009).

16. Holmes, D. P. Elasticity and stability of shape-shifting structures. Curr. opinion colloid \& interface science 40, 118-137 (2019).

17. Shyer, A. E. et al. Villification: how the gut gets its villi. Science 342, 212-218 (2013).

18. Garcia, K., Kroenke, C. \& Bayly, P. Mechanics of cortical folding: stress, growth and stability. Philos. Transactions Royal Soc. B: Biol. Sci. 373, 20170321 (2018).

19. Mandaravally Madhavan, M. \& Schneiderman, H. A. Histological analysis of the dynamics of growth of imaginal discs and histoblast nests during the larval development of Drosophila melanogaster. Wilhelm Roux's Arch. Dev. Biol. 183, 269-305, 10.1007/BF00848459 (1977). 
20. Lawrence, P. A., Green, S. M. \& Johnston, P. Compartmentalization and growth of the Drosophila abdomen. J. Embryol. Exp. Morphol. Vol. 43, 233-245, 10.1242/dev.43.1.233 (1978).

21. Bakker, K. Feeding period, growth, and pupation in larvae of drosophila melanogaster. Entomol. Exp. et Appl. 2, 171-186 (1994).

22. Harris, T. J. \& Tepass, U. Adherens junctions: from molecules to morphogenesis. Nat. reviews Mol. cell biology 11, 502-514 (2010).

23. Brüser, L. \& Bogdan, S. Adherens junctions on the move-membrane trafficking of e-cadherin. Cold Spring Harb. perspectives biology $\mathbf{9}$, a029140 (2017).

24. Ninov, N. \& Martín-Blanco, E. Changing gears in the cell cycle: histoblasts and beyond. Fly 3, 286-289, 10.4161/fly.10443 (2009).

25. Sumi, A. et al. Adherens junction length during tissue contraction is controlled by the mechanosensitive activity of actomyosin and junctional recycling. Dev. cell 47, 453-463 (2018).

26. Kakanj, P. et al. Insulin and TOR signal in parallel through FOXO and S6K to promote epithelial wound healing. Nat. Commun. 7, 10.1038/ncomms12972 (2016).

27. Yonemura, S. Cadherin-actin interactions at adherens junctions. Curr. opinion cell biology 23, 515-522 (2011).

28. Lopata, A. et al. Affimer proteins for f-actin: novel affinity reagents that label f-actin in live and fixed cells. Sci. reports $\mathbf{8}$, $1-15$ (2018).

29. LeGoff, L., Rouault, H. \& Lecuit, T. A global pattern of mechanical stress polarizes cell divisions and cell shape in the growing drosophila wing disc. Development 140, 4051-4059 (2013).

30. Mao, Y. et al. Differential proliferation rates generate patterns of mechanical tension that orient tissue growth. EMBO J. 32, 2790-2803, 10.1038/emboj.2013.197 (2013).

31. Rauskolb, C., Sun, S., Sun, G., Pan, Y. \& Irvine, K. D. Cytoskeletal tension inhibits hippo signaling through an ajuba-warts complex. Cell 158, 143-156 (2014).

32. Biglou, S. G., Bendena, W. G. \& Chin-Sang, I. An overview of the insulin signaling pathway in model organisms drosophila melanogaster and caenorhabditis elegans. Peptides 145, 170640 (2021).

33. Woichansky, I., Beretta, C. A., Berns, N. \& Riechmann, V. Three mechanisms control e-cadherin localization to the zonula adherens. Nat. communications 7, 1-11 (2016).

34. Edgar, B. A., Sprenger, F., Duronio, R. J., Leopold, P. \& O'Farrell, P. H. Distinct molecular mechanism regulate cell cycle timing at successive stages of drosophila embryogenesis. Genes \& development 8, 440-452 (1994).

35. Neufeld, T. P. \& Edgar, B. A. Connections between growth and the cell cycle. Curr. Opin. Cell Biol. 10, 784-790, 10.1016/S0955-0674(98)80122-1 (1998).

36. Brangwynne, C. P. et al. Microtubules can bear enhanced compressive loads in living cells because of lateral reinforcement. The J. cell biology 173, 733-741 (2006).

37. Podewitz, N., Delarue, M. \& Elgeti, J. Tissue homeostasis: A tensile state. Epl 109, 10.1209/0295-5075/109/58005 (2015).

38. Priya, R. et al. Feedback regulation through myosin II confers robustness on RhoA signalling at E-cadherin junctions. Nat. Cell Biol. 17, 1282-1293, 10.1038/ncb3239 (2015).

39. Bischoff, M. \& Cseresnyés, Z. Cell rearrangements, cell divisions and cell death in a migrating epithelial sheet in the abdomen of Drosophila. Development 136, 2403-2411, 10.1242/dev.035410 (2009).

40. David, D. J., Tishkina, A. \& Harris, T. J. The PAR complex regulates pulsed actomyosin contractions during amnioserosa apical constriction in Drosophila. Development 137, 1645-1655, 10.1242/dev.044107 (2010).

41. Jayasinghe, A. K., Crews, S. M., Mashburn, D. N. \& Hutson, M. S. Apical oscillations in amnioserosa cells: Basolateral coupling and mechanical autonomy. Biophys. J. 105, 255-265, 10.1016/j.bpj.2013.05.027 (2013).

42. Sawyer, J. M. et al. Apical constriction: a cell shape change that can drive morphogenesis. Dev. biology 341, 5-19 (2010).

43. Sapala, A. et al. Why plants make puzzle cells, and how their shape emerges. Elife 7, e32794 (2018).

44. Carter, R., Sánchez-Corrales, Y. E., Hartley, M., Grieneisen, V. A. \& Marée, A. F. Pavement cells and the topology puzzle. Development 144, 4386-4397 (2017).

45. Valon, L. \& Levayer, R. Dying under pressure: cellular characterisation and in vivo functions of cell death induced by compaction. Biol. Cell 111, 51-66 (2019). 
46. García-Bellido, A. \& Merriam, J. R. Parameters of the wing imaginal disc development ofdrosophila melanogaster. Dev. biology 24, 61-87 (1971).

47. Heemskerk, I., Lecuit, T. \& LeGoff, L. Dynamic clonal analysis based on chronic in vivo imaging allows multiscale quantification of growth in the drosophila wing disc. Development 141, 2339-2348 (2014).

48. Meng, H., Nuzhding, D., Sison, M., Frédéric \& LeGoff, L. Adaptive scans allow targeted cell-ablations on curved cell sheets. BioRxiv https://doi.org/10.1101/2022.01.30.478374 (2022).

49. Berg, S. et al. ilastik: interactive machine learning for (bio)image analysis. Nat. Methods 10.1038/s41592-019-0582-9 (2019).

50. Abouakil, F. et al. An adaptive microscope for the imaging of biological surfaces. Light. Sci. \& Appl. 10, 1-12 (2021).

51. Aigouy, B., Umetsu, D. \& Eaton, S. Segmentation and quantitative analysis of epithelial tissues. Drosophila 227-239 (2016). 


\section{Supplementary material}
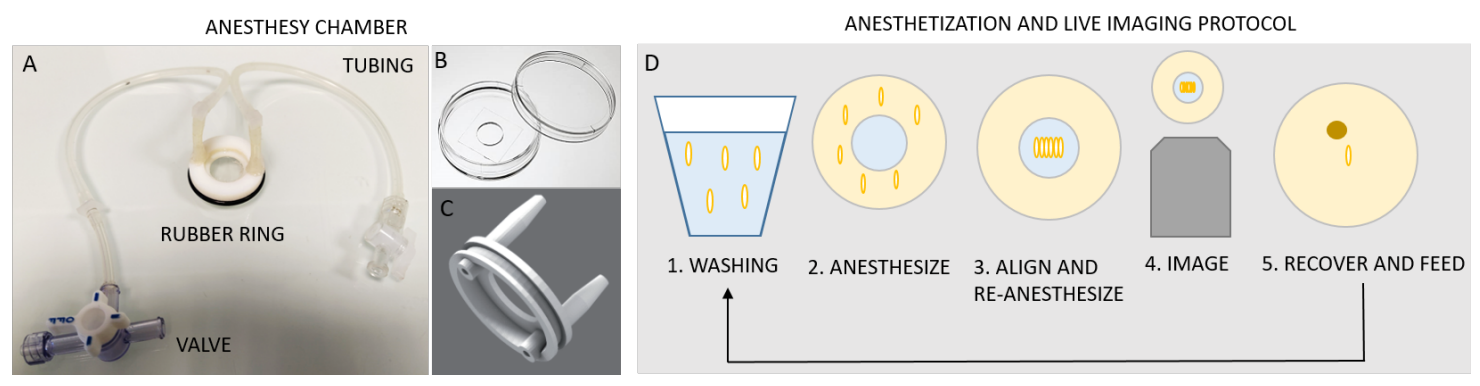

Figure S1. Anesthesy chamber and imaging protocol A: Assembled anesthesy chamber made of a glass-bottom dish (zoom in B), a custom-designed injection lid (zoom in C), tubing and valves to which syringes are then connected. D: Main steps of the imaging protocol: 1) larvae are washed in PBS and dried on a lab wipe 2) Larvae are positioned around the glass of the Petri dish and anesthetized for $5 \mathrm{~min}$; 3) After closing the valves to keep the anesthetic, larve are aligned on the glass, with a drop of halocarbon oil; 4) Larvae are images by confocal spinning disc microscopy; 5) For chronic imaging, each larva is placed in a petri dish with soft food and let recover for a few hours before starting the procedure at 1. 

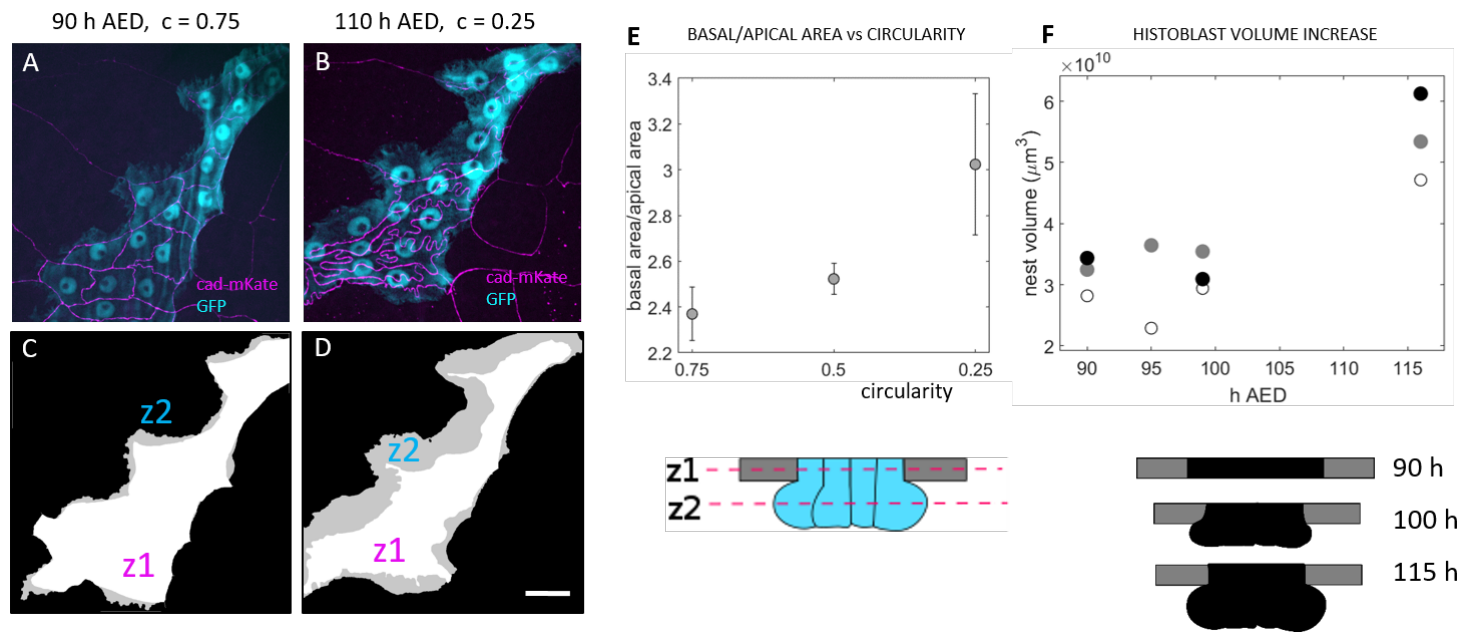

Figure S2. 3D Characterization of histoblast growth A - Z-projection of cad:mKate (magenta) and cytosolic GFP (cyan) taken in a larva at $90 \mathrm{~h} \mathrm{AEL}$, i.e. at the beginning of the buckling transition. B Z-projections of the same larva 20 hours later, after the formation of junctional folds. C,D Masks of the external contour of the apical and basal sides of the larva in A. The white area is obtained from the apical plane obtained from cad:mkate ( $\mathrm{z} 1$ in the schematic representation), the gray area from the cytosolic GFP maximum projections. It corresponds to the basal plane at which the nest is the largest ( $\mathrm{z} 2$ in the schematic represetations). Before the transition (C) z1 and z2 are almost superposed, while after transition (D) z2 is much bigger than z1, meaning that HBs expand basally. E Ratio of basal/apical area for different circularity values, obtained from . As HBs junctions fold, the basal areal becomes larger than the apical. Apical and basal areas correspond to the the adherens region and the largest basal area, as schmetized below the plot. F Total nest volume at different times. White, gray and black dots correspond each to one histoblasts nest. As apical surface shrink, histoblasts expand below the adherens region, as schematized below the plot. 

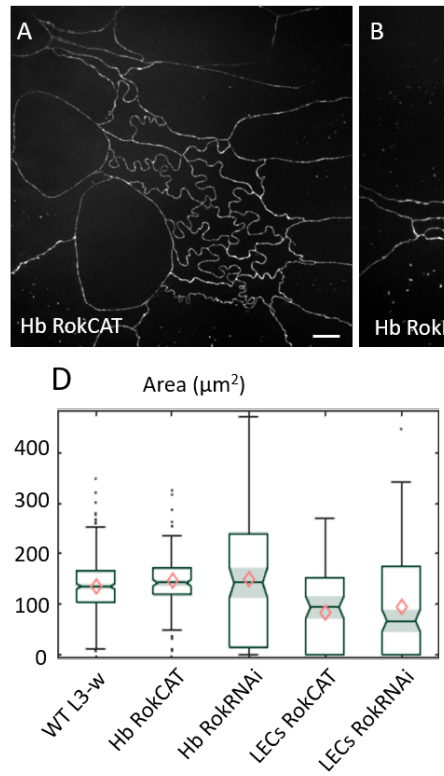
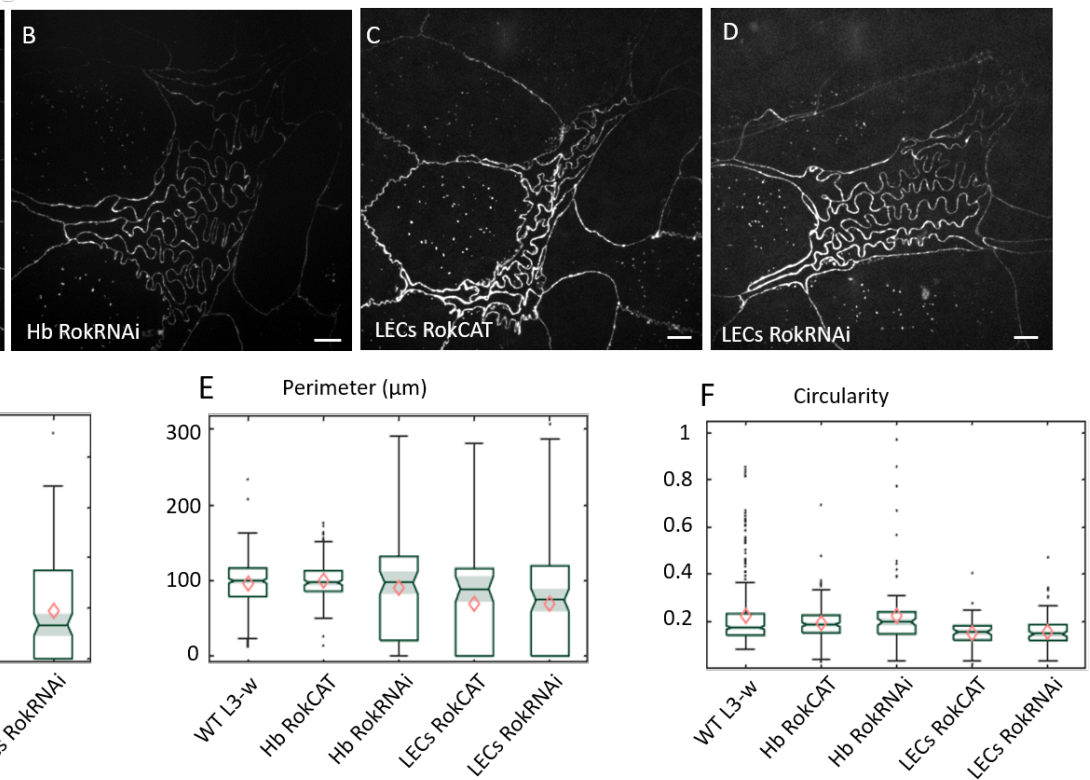

Figure S3. Genetic perturbation of cell contractility All images were taken from living larvae at the wondering stage. A: $H b$ RokCAT = mutant histoblasts in a constitutively active form of Rok was expressed. B: Hb RokRNAi= mutant histoblasts in which an RNAi against Rok was expressed. A: LECs RokCAT = mutant flies in which a constitutively active form of Rok was expressed in LECs. D: LECs RokRNAi = mutant LECs in which an RNAi against Rok was expressed. F-H Boxplots of cell area, perimeter and circularity for the different cases illustrated above. $\mathrm{WT}=$ wilde type. $\mathrm{N}=352,176,138,115,158$ cells for each data set, ordered as in the figure.

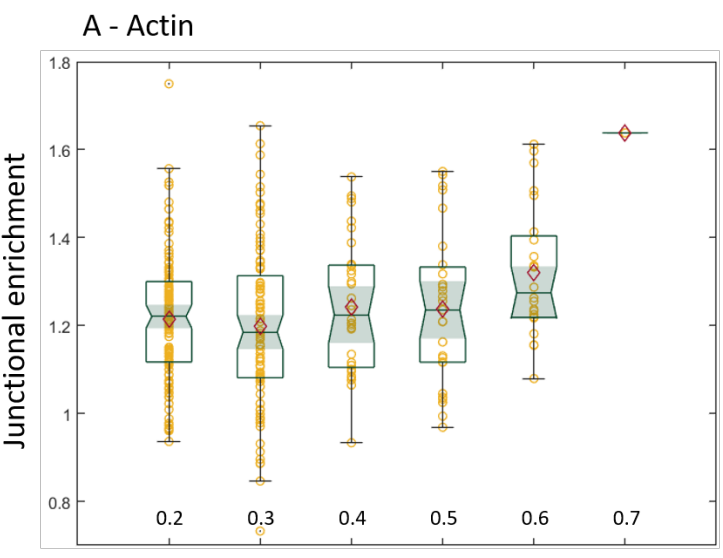

Cell circularity

\section{B - Myosin}

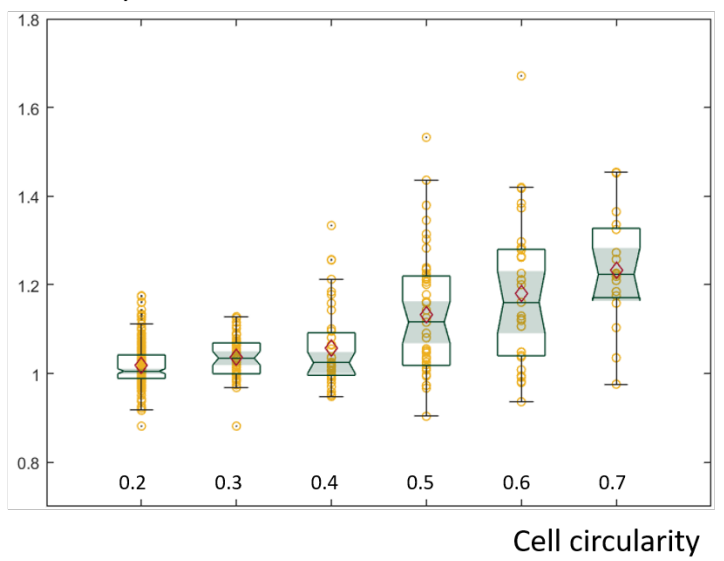

Figure S4. Junctional enrichment of actin and myosin Plot of relative amount of junctional actin (A) and myosin (B) as a function of circularity, calculated as the ratio junctional signal/cystosolic signal at the same plane. The data were binned with the same bin size for both actin and myosin. The horizontal bar represents the median for each bean, the shadowed areas the confidence interval of 0.05 , the diamonds correspond to the mean value for each bin and the yellow circles are single data points. Pearson's correlation coefficients calculated on all the data where 0.18 with a p-value of 0.002 for actin, and of 0.59 with p-value $1^{-32}$ for myosin data. T-test comparisons for the junctional enrichement of the first and last point gave p-values of 0.002 and 1.39e-33, for actin and myosin respectively. For each bin, $\mathrm{N}=116,88,32,2724,1$ (actin) and $\mathrm{N}=199,50,39,45$, 29,17 
bioRxiv preprint doi: https://doi.org/10.1101/2022.02.10.479869; this version posted February 10, 2022. The copyright holder for this preprint (which was not certified by peer review) is the author/funder. All rights reserved. No reuse allowed without permission.

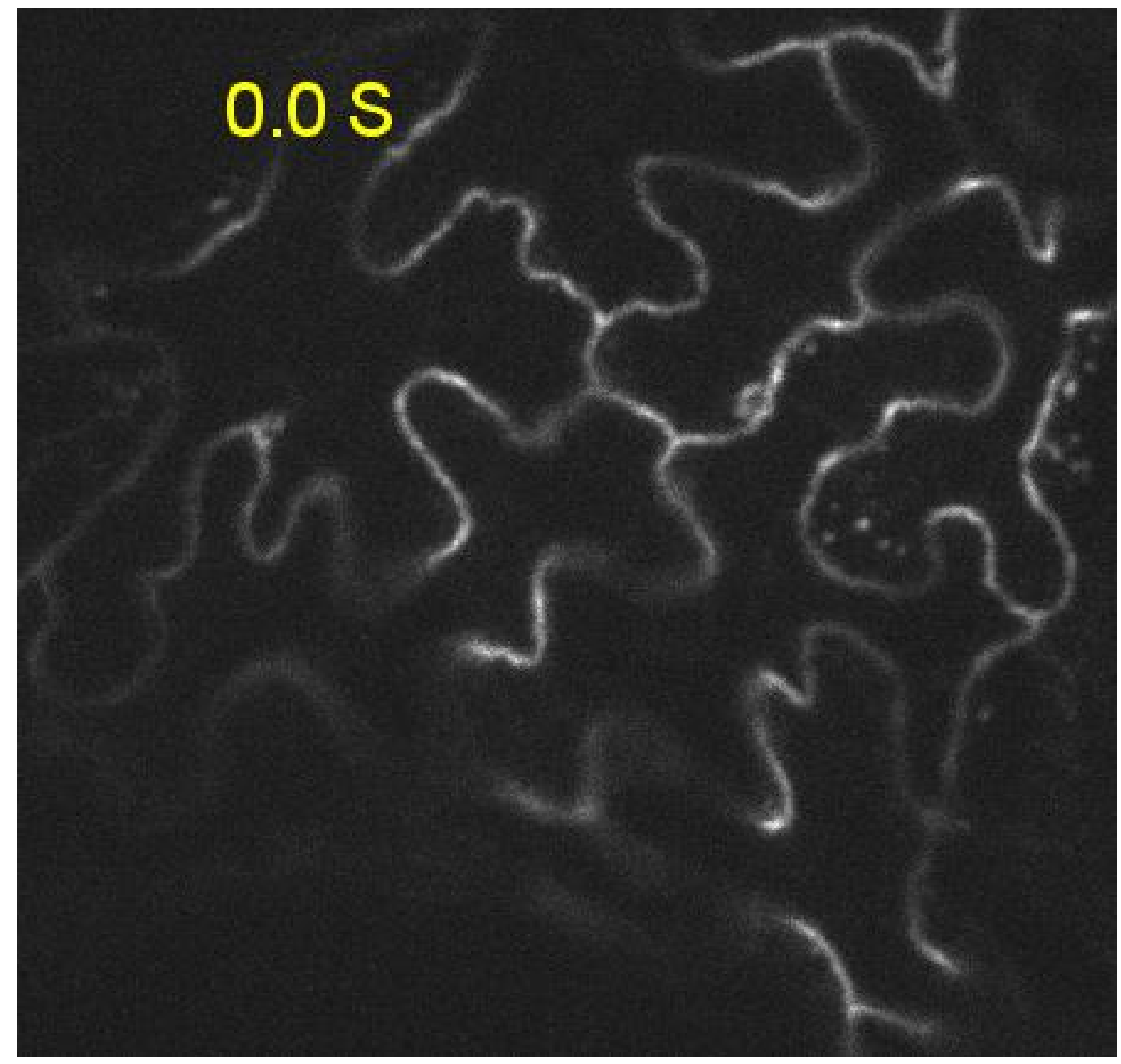

Figure S5. Movie S1 - Laser ablation of folded histoblasts 
bioRxiv preprint doi: https://doi.org/10.1101/2022.02.10.479869; this version posted February 10, 2022. The copyright holder for this preprint (which was not certified by peer review) is the author/funder. All rights reserved. No reuse allowed without permission.

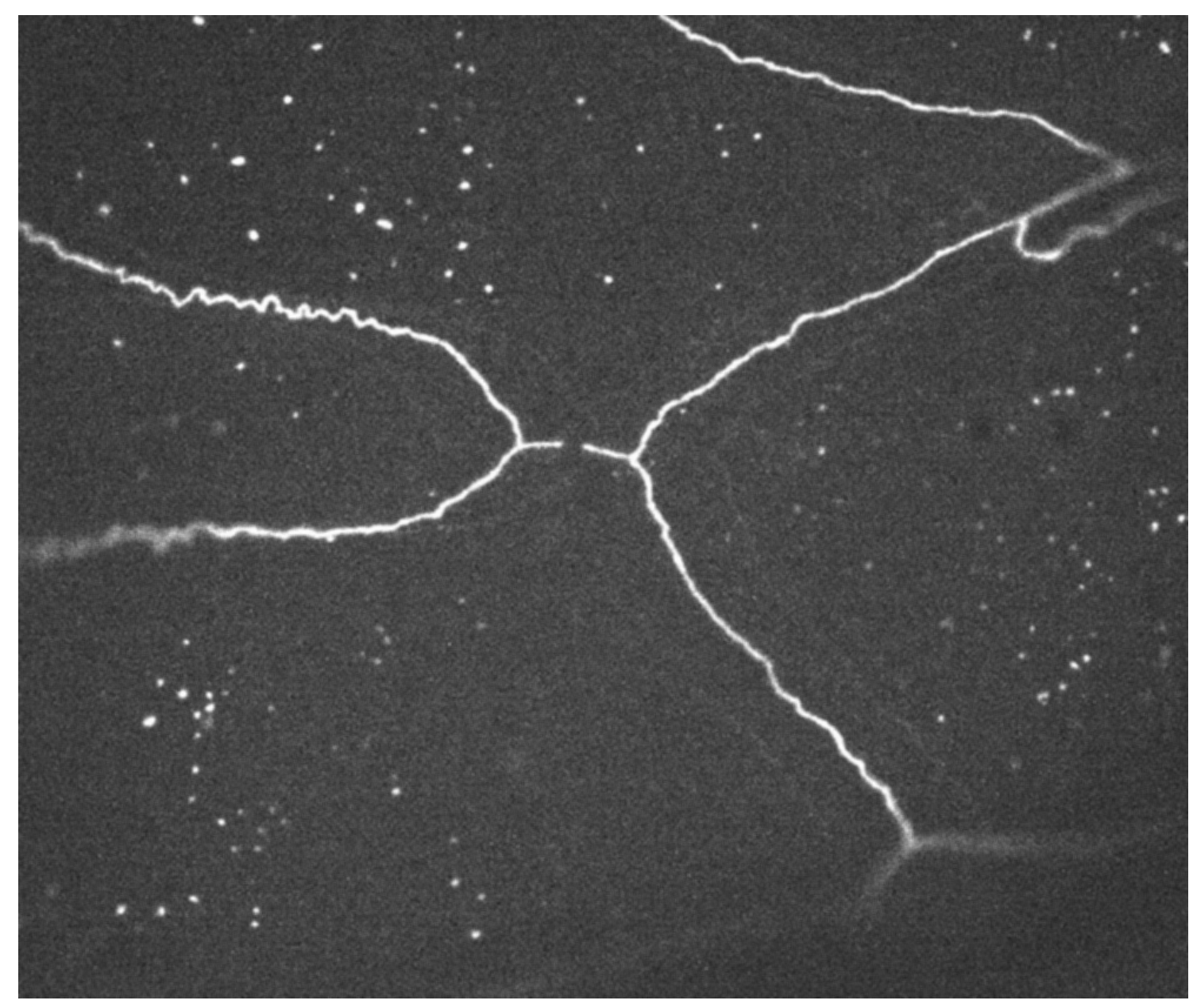

Figure S6. Movie S2 - Laser ablation of LECs close to histoblasts 